\title{
Walk-off Induced Dissipative Quadratic Solitons in Degenerate Optical Parametric Oscillators
}

\author{
Arkadev Roy ${ }^{1}$, Rajveer Nehra ${ }^{1}$, Saman Jahani $^{1}$, Luis Ledezma ${ }^{1}$, Carsten Langrock ${ }^{2}$, \\ Martin Fejer ${ }^{2}$, Alireza Marandi ${ }^{1}$ \\ ${ }^{1}$ Electrical Engineering Department, California Institute of Technology, Pasadena, California, 91125, USA \\ ${ }^{2}$ Edward L. Ginzton Laboratory, Stanford University, Stanford, California, 94305, USA \\ marandi@caltech.edu
}

\begin{abstract}
We demonstrate quadratic soliton formation in a synchronously pumped degenerate optical parametric oscillator displaying significant pulse compression leading to the formation of femtosecond pulses at half-harmonic from picosecond pump pulses.(C) 2021 The Author(s)
\end{abstract}

Formation of dissipative solitons in nonlinear resonators has become a versatile mechanism for stable femtosecond sources [1]. Kerr frequency combs in micro-resonators have been successful in accessing these soliton regimes in a compact footprint [1]. However, so far most of these devices leverages the cubic (Kerr) nonlinearity which is only capable of generating solitons centered around the pump frequency. In contrast, devices utilizing the secondorder (quadratic) nonlinearity can efficiently generate frequency combs with octave separations (second-harmonic/ half-harmonic), thereby paving the way for frequency comb sources in less-accessible wavelength ranges such as visible, UV, as well as mid-IR [2,3]. Here, we present synchronously pumped quadratic soliton formation at the half-harmonic of a narrow-band pump in a degenerate optical parametric oscillator (OPO).

(a)
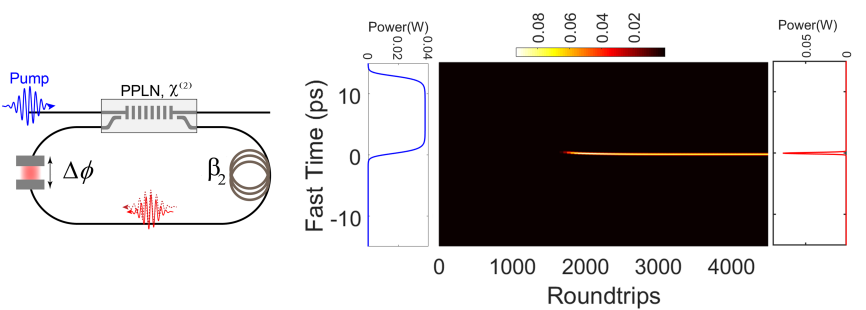

(c)

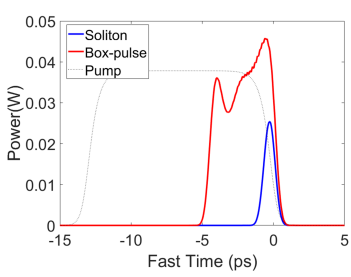

(d)

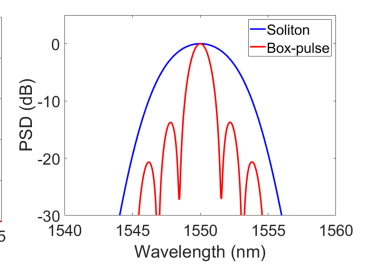

Fig. 1. a) Schematic of the OPO comprising of the PPLN waveguide, fiber-feedback cavity circulating the resonant signal/idler and pumped by picosecond pulses at $775 \mathrm{~nm}$. b) Numerical results of soliton formation (roundtrip evolution) shown as the OPO reaches the steady state forming utra-short signal at half-harmonic starting from ps long pump. Operation of the OPO in two regimes, namely the soliton regime (near-threshold), and box-pulse regime (far from threshold). Typical temporal (c) and spectral (d) characteristics are shown as obtained from numerical simulation.

We consider a doubly-resonant OPO, where the signal and the idler are resonant in a fiber feedback cavity as shown in Fig. 1(a). The quadratic soliton formation takes place in the presence of temporal group-velocity mismatch $(\mathrm{GVM})$. The group velocity of the signal is larger than the pump (for the frequencies under consideration) and is estimated to be $\sim 0.329 \mathrm{ps} / \mathrm{mm}$. The signal soliton walks through the pump and extracts parametric gain in the process. The optimum pump length for efficient pump conversion (in terms of pulse compression) has to be matched to the walk-off length of the periodically-poled lithium niobate (PPLN) crystal (i.e. $T_{p}=L u$, where $T_{p}, L$, and $u$ are the pump pulse length, length of the crystal, and group velocity mismatch respectively.) The dissipative soliton is sustained by energy balance, where the parametric gain counteracts the round-trip dissipation (propagation loss + out-coupling loss). The chirp is balanced by the interplay between dispersion and gain-clipping, while the pulse timing balance is ensured by the mutual dynamics involving cavity detuning, gain-saturation induced nonlinear interaction, and gain-clipping. Variational calculations reveal that the quadratic soliton projected on a hyperbolic secant ansatz assume a pulse width which is given by: $\tau_{p}=\left[\frac{7}{15} \frac{\beta_{2}^{2} T_{p}}{\ln \left(G \mathrm{e}^{\alpha L}\right) \ln (2)}\right]^{\frac{1}{5}}$, where $G$ is the power gain at threshold, $\alpha$ is the propagation loss per unit length, $\beta_{2}$ is the total integrated dispersion [4]. The pulse compression ratio is then obtained as $\frac{T_{p}}{\tau_{p}}$. The pulse shaping mechanism is driven by the temporal walk-off due to the GVM, and solitons can form both in normal and anomalous 
dispersion regime. The GVM enabled soliton formation mechanism is in stark contrast to simulton (locked bright signal and dark pump) pulse shaping mechanism also occurring in degenerate OPOs [5]. Figure 1(b) shows the roundtrip intra-cavity intensity evolution depicting the soliton formation in the steady state. It is to be noted that the OPO under consideration can also support non-degenerate oscillation and spectral phase transitions [6].

In the experiment a PPLN waveguide (40 mm long) provides parametric interaction between $775 \mathrm{~nm}$ pump and $1550 \mathrm{~nm}$ signal at the half-harmonic [7]. Intra-cavity group velocity dispersion $\left(\beta_{2}\right)$ management is done by a combination of standard and dispersion compensating polarization maintaining fibers. The OPO is synchronously pumped by pump pulses approximately $13 \mathrm{ps}$ long. The cavity delay $(\Delta \phi)$ is adjusted to match the repetition rate of the synchronous pumping to an integer multiple of the cavity FSR.

(a)

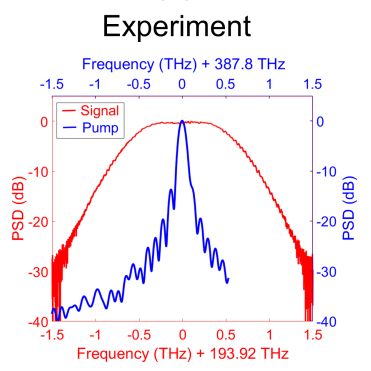

(b)

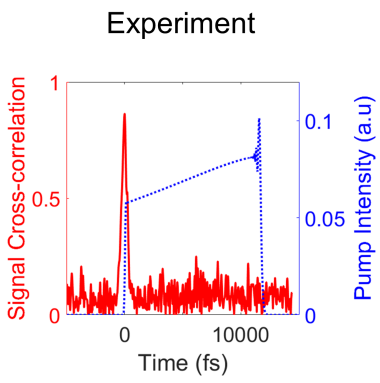

(c)

Experiment

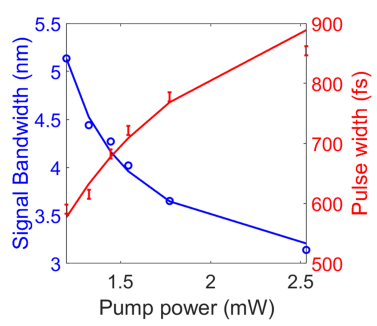

(d)

Simulation

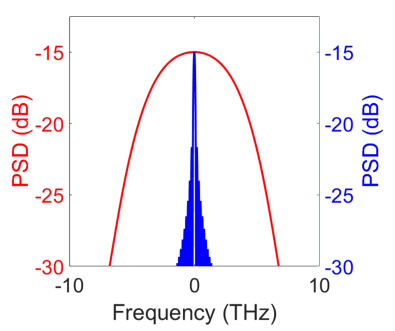

Fig. 2. a) Spectrum of the quadratic soliton (red curve) exhibiting significant broadening as compared to the narrowband pump (blue curve). b) Cross-correlation trace showing the temporal intensity profile of the soliton (red curve) depicting significant pulse compression compared to the ps long pump pulse (blue dotted curve). c) Power scaling properties of the quadratic soliton. The bandwidth (blue curve) gets narrower with a corresponding widening of the pulse temporal width (red curve) as the pump power is ramped above threshold. d) Giant pulse compression as expected from a dispersion engineered thin-film waveguide based OPO obtained using numerical simulation.

Figure 2(a) shows the spectral characteristics of the signal ( $7.5 \mathrm{~nm} 3 \mathrm{~dB}$ bandwidth) and the pump which amounts to $\sim 40$ times broadening. The corresponding temporal domain compression is shown in Fig. 2(b). The temporal profile is measured using cross-correlation (non-collinear sum-frequency generation) with ultra-short $(\sim 40 \mathrm{fs})$ local oscillator. We measured soliton temporal width of $\sim 540 \mathrm{fs}$, which can be further compressed via chirp compensation to its transform-limited value. The power scaling behavior is displayed in Fig. 2(c) which shows a spectral narrowing with increasing pump power above threshold as the soliton transits into a box-pulse regime [4]. The box pulse regime occurs for OPO away from threshold and is dominated by gain-clipping and gain-saturation, with the cavity dispersion playing a minor role. Distinct behavior of the OPO operating in the soliton and boxpulse regimes is shown in Fig. 1(c,d). The degenerate operation of the OPO is confirmed from the radio-frequency beat-note measurement with the femtosecond local oscillator.

We have shown that in the presence of large temporal walk-off it is possible to obtain significant temporal compression and sustain a dissipative soliton in a synchronously pumped degenerate OPO. Further improvement is possible by precise dispersion control. With the recent advancements in integrated lithium niobate device fabrication, it is possible to engineer the group-velocity dispersion and temporal walk-off, thereby optimizing the figure of merit. Numerical simulations suggest that pulse-compression in excess of a factor of 125 is feasible as shown in Fig. 2(d). Desirable pulse scaling behavior can be achieved if operated in the simulton regime [5].

This work is supported by AFOSR award FA9550-20-1-0040, NSF Grant No. 1846273, and NASA.

\section{References}

1. T. Kippenberg, A. Gaeta, M. Lipson, M. Gorodetsky Science 361(6402), (2018).

2. A. Bruch, X. Liu, Z. Gong, J. Surya, M. Li, CL Zou, H. Tang, Nat. Photonics, , (2020).

3. S. Mosca, M. Ricciardi, M. Parisi, L. Maddaloni, P. San-tamaria, M. Rosa, Nanophotonics, 5(316), (2016).

4. R. Hamerly, A. Marandi, M. Jankowski, M. Fejer, Y. Yamamoto and H. Mabuchi, PRA 94(6), (2016).

5. M. Jankowski, A. Marandi, CR Phillips, R. Hamerly, K. Ingold, R. Byer, MM Fejer, PRL, 120(5), (2018).

6. A. Roy, S. Jahani, C. Langrock, M. Fejer, A. Marandi, arXiv:2009.00930 (2020).

7. C. Langrock, and M.M. Fejer, Optics letters 32(15) , (2007). 